Faculdade de Ciências Econômicas UFRGS
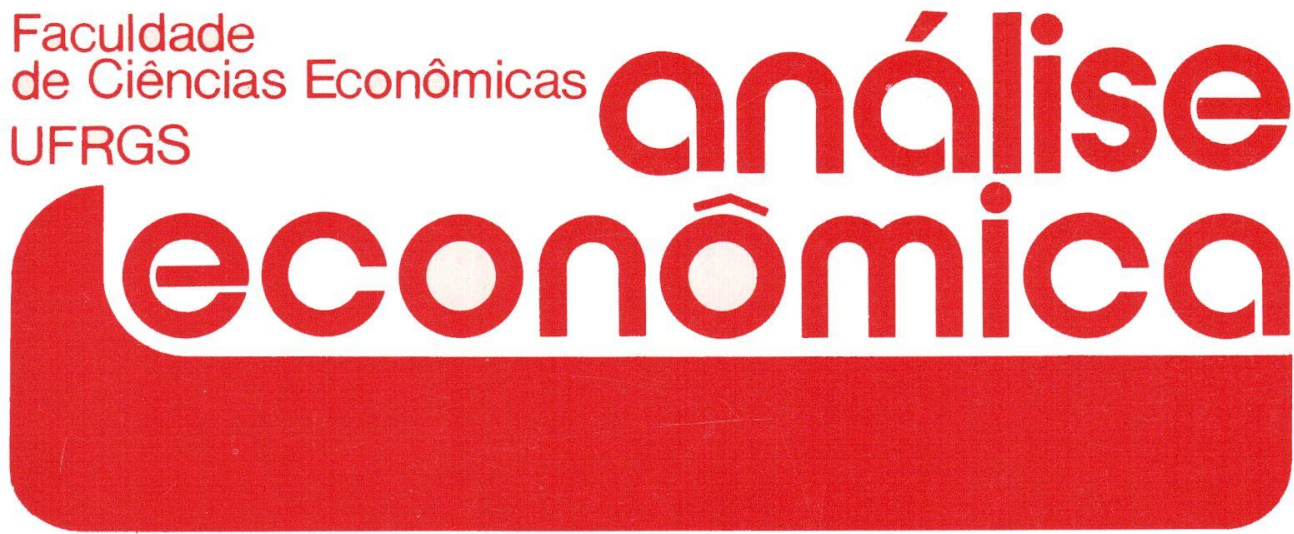

- INDEXAÇĀo SALARIAL: UMA ABORDAGEM MACROECONÓMICA Jo Anna Grav

- AJUSTE NO EMPREGO E PRODUTIVIDADE NA DECADA DE OITENTA

Carlos Antônio Luque José Paulo Zeeteno Chahad

- O CONSUMIDOR KEYNESIANO Marcelo Córtes Neri

- HETEROGENEIDADE DO TRABALHO E TAXA DE LUCRO EM MARX

Francisco Cribari Neto

- ECONOMIAS DE ESCALA: UMA REVISĀO Jesiel de Marco Gomes

- Concentraçấo bancária no BRASIL

Marcelo Resende

- NOYOS RUMOS PARA O SETOR ELETRICO NO BRASIL

Adriano Pires Rodrigues

Eduardo da Cunha Vianna

- OFERTA E DEMANDA DE FRANGO DE CORTE NO BRASIL

Narciso Gonçalves de Castro et alii

- ANÁLISE ECONÔMICA DA IRRIGAÇÃo DO MILHO

Lúcia M Schirmer

Juvir Luiz Mattuella

- REFLORESTAMENTO NO BRASIL. Carlos José Caetano Bacha

- ESCOLHA DE TECNOLOGIA EM ESTRUTURA DE PRINCIPAL AGENTE Kyle D. Kauffma:

- A QUESTÁo DEMOGRÁfica e A PRAXEOLOGIA

Anton Karl Biedermann et alii
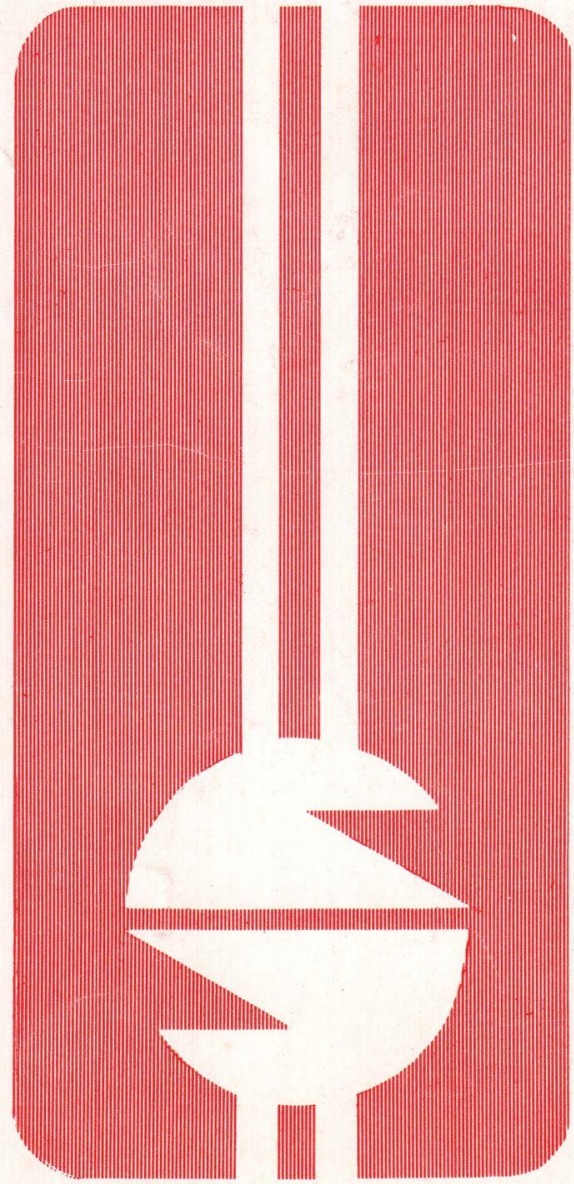
UNIVERSIDADE FEDERAL DO RIO GRANDE DO SUL

Reitor: Prof. Tuiskon Dick

FACULDADE DE CIÉNCIAS ECONÔMICAS

Diretora: Prof ${ }^{\text {a }}$ Yeda Rorato Crusius.

CENTRO DE ESTUDOS E PESQUISAS ECONÔMICAS

Diretor: Reinaldo Ignacio Adams

DEPARTAMENTO DE CIÉNCIAS ECONÔMICAS

Chefe: Prof. Fernando Ferrari Filho

CURSO DE POS-GRADUAÇÄO EM ECONOMIA

Coordenador: Prof. Nali de Jesus de Souza

CURSO DE PÓS-GRADUAÇĀO EM ECONOMIA RURAL

Coordenador: Prof. Atos Freitas Grawunder

CONSELHO EDITORIAL: Achyles Barcelos da Costa, Aray Miguel Feldens, Atos Freitas Grawunder, Carlos Augusto Crusius, Ernani Hickmann, João Rogério Sanson, Juvir Luiz Mattuella, Maria Imilda da Costa e Silva, Nali de Jesus de Souza, Nuno Renan Lopes de Figueiredo Pinto, Otilia Beatriz Kroeff Carrion, Otto Guilherme Konzen, Paulo Alexandre Spohr, Pedro Cezar Dutra Fonseca, Reinaldo Ignacio Adams, Roberto Camps Moraes, Valter José Stülp, Yeda Rorato Crusius, David Garlow (Wharton Econometrics Forecasts Association, E.U.A.), Edgar Augusto Lanzer (UFSC), Eleutério F.S. Prado (USF), Fernando Holanda Barbosa (FGV/RJ), Gustavo Franco (PUC/RJ), Joaquim Pinto de Andrade (UnB), Juan H. Moldau (USP), Werner Baer (Univ. de Illinois, E.U.A.).

COMISSĀO EDITORIAL: Atos Freitas Grawunder, Pedro Cezar Dutra Fonseca, Reinaldo Ignacio Adams e Roberto Camps Moraes.

EDITOR: Nali de Jesus de Souza

SECRETARIA: Maria Ivone de Mello (normalização), Vanete Ricacheski (revisão de textos).

FUNDADOR: Prof. Antônio Carlos Santos Rosa

Os materiais publicados na revista Análise Econômica são de exclusiva responsabilidade dos autores. É permitida a reprodução total ou parcial dos trabalhos, desde que seja citada a fonte.

Aceita-se permuta com revistas congêneres. Aceitam-se, também, livros para divulgação, elaboração de resenhas ou recenșões.

Toda correspondência, material para publicação, assinaturas e - permutas devem ser dirigidos ao seguinte destinatário:

\section{PROF. NALI DE JESUS DE SOUZA}

Revista Análise Econômica

Av. João Pessoa, 52

CEP 90040-000 - PORTO ALEGRE (RS), BRASIL

Telefones: (051) 228-1633 - 224-6024 ramais 3440 e 3348

Fax: (051) 225-1067 


\title{
OFERTA E DEMANDA DE FRANGO DE CORTE NO BRASIL
}

\author{
Narciso Gonçalves de Castro ${ }^{2}$ \\ Ricardo Pereira Reis ${ }^{3}$ \\ Antonio João dos Reis ${ }^{3}$ \\ Arnaldo Pereira Vieira ${ }^{3}$
}

\section{SINOPSE}

Este trabalho teve como objetivo geral o estudo da oferta e demanda de frango de corte no Brasil. Foram utilizados dados de séries temporais referentes ao perfodo de 1972 e 1988 e o modelo analítico baseou-se numa função do tipo Cobb-Douglas. $\mathrm{O}$ modelo cconométrico para oferta e demanda foi desenvolvido com base num sistema de equaçoses simultaneas e os parâmetros foram estimados com base nos mínimos quadrados em dois estágios (MQ2E). Os principais resultados encontrados foram os seguintes: A elasticidade-preço da oferta de frango de corte $e$ inelástica, enquanto que a da demanda é elástica; os avicultores brasileiros parecem ser mais sensíveis a aumentos nos preços do frango do que a redução nos preços da raçấo balanceada; as polf́ticas de congelamento de preços implementadas no Brasil têm se mostrado ineficientes diante de demandas elásticas; constatou-se uma relaçăo de substituiçăo entre a carne de frango e as carnes bovina e suina.

\section{INTRODUÇÃO}

Um dos problemas que mais preocupa os países em desenvolvimento é a relação entre o crescimento populacional e a produção de alimentos.

Segundu Teixeirá (1970) e Marra et aliii (1990), a produção de alimentos é uma das atividades econômicas de maior importância para a humanidade, principalmente se se levar em consideração que as previsões sobre o crescente aumento populacional indicam 179,5 milhốes de habitantes no Brasil e 7,3 bilhões de habitantes no mundo, a serem ali-

1 Parte da dissertação apresentada à Escola Superior de Agricultura de Lavras (ESAL), pelo
primeiro autor, para obtenção do grau de Mestre em Administração Rural.
2 Engenheiro Agrónomo com Mestrado em Administração Rural e Assistente TÉcnico da
EMATER/RS na Área de Administração Rural. Caixa Postal 2727, 90060 - Porto Alegre -
RS.
Professores do Departamento de Administração e Economia da Escola Superior de Agricul-
tura de Lavras - LAVRAS - MG.
\begin{tabular}{|c|c|c|c|c|}
\hline ANALISE ECONÔMICA & ANO 10 & NNo 17 & MARÇO/92 & P.117-126 \\
\hline
\end{tabular} 
mentados no ano 2.000 .

Nos úlimos 20 anos, a economia brasileira vem passando por uma fase particularmente crf́tica, talvez a pior crise econômica de sua história, configurada num sério problema externo, representado por elevada dfvida externa e de um problema interno angustiante, figurado nas altas taxas de inflação e no agravamento das desigualdades sociais.

Apesar desses problemas apresentarem reflexos negativos em todos os segmentos da economia, será dada, no presente estudo, atenção ao setor de alimentos e, em particular, aos aspectos ligados à oferta e à demanda de frango de corte e o seu inter-relacionamento com as carnes bovina e sulna, que se constituem numa importante fonte supridora de proteína para a população brasileira.

Conforme Fernandes et alii (1981), o desenvolvimento alcançado pela avicultura nacional colocou a atividade em posição privilegiada em relação a outras explorações animais, já que detém um dos maiores acervos tecnológicos do setor agropecuário. De acordo com a FAO (Food and Agriculture Organization), à avicultura é a exploração zootécnica capaz de colaborar positivamente na minimização dos graves problemas de alimentação da crescente população mundial.

A avicultura brasileira tem alcançado alto nf́vel tecnológico, empregando uma tecnologia intensiva em capital e terra, quando comparada com outras explorações agrícolas, Barrios (1981). De acordo com a SADIA (s. d.), a produtividade da avicultura nacional vem apresentando ganhos significativos. Em regra geral, isto significa que os custos totais de produção devem aumentar à medida que se investir em modernização.

$O$ valor econômico e social da atual indústria avícola brasileira é expressivo, especialmente levando-se em conta que ela movimenta uma série de atividades correlatas, bem como atividades de intermediação na conırcialização, beneficiamento e prestação de serviços de seus produtos.

A avicultura é um setor de grande dinamismo e de importância econômico-social dentro do contexto da agropecuária nacional, gerando cerca de um milhão de empregos diretos através de granjas, abatedouros e indústrias, sem considerar os criados pelas empresas de atividades correlatas. De acordo com Englert (1974/78) e Informes GEP/DESR (1990), o consumo per capita de frango tem aumentado de forma constante no mercado interno, passando de $3,3 \mathrm{~kg} / \mathrm{hab}$ em 1974, para 6,6 $\mathrm{kg} / \mathrm{hab}$ em 1977, 11,8 kg/hab em 1988, 12,5 kg/hab em 1989 e cerca de $14,5 \mathrm{~kg} / \mathrm{hab}$ em 1990 . E, em parte, por manter preços históricos inferiores as outras carnes, principalmente à bovina, a carne de frango conquistou parcelas significativas do mercado, antes dominadas pelas outras carnes. Percebe-se, assim, que a carne de frango, ano a ano, aumenta a sua participação no cardápio da população brasileira, "tomando" espaço 
da carne bovina, apesar de sua demanda regionalizada, pois conforme dados da ANAB (s. d.), em 1984, cerca de $91 \%$ dos abates no Pafs foram realizados na região Sul e no estado de São Paulo.

$O$ crescimento da contribuição da avicultura no PIB, durante o perfodo 1972-88 foi sempre superior ao crescimento da produção brasileira agregada, a exceção dos anos de 1984, 1985 e 1988 (anos de crise no setor avícola). De 1972 a 1988, a avicultura nacional teve um crescimento médio anual de $9,6 \%$, enquanto o crescimento do Pafs situou-se em torno de $2,0 \%$.

Com o crescimento da população brasileira, cresce também a demanda por carnes, contrastando com o poder aquisitivo do povo brasileiro, cuja renda per capita, apesar de ter aumentado na decada de 70 , manteve-se declinante nos anos 80 . Dessa forma, a população precisa buscar alternativas mais baratas para adquirir o produto. Como o preço da carne de frango é cerca de $70 \%$ do preço da carne bovina, Englert (1978) prevê que a produção de carne de aves poderá aumentar, pelo menos cinco vezes, sem que se verifique uma saturação do mercado nacional.

Eis ar um problema desafiante para a avicultura nacional: produzir o suficiente para atender a crescente demanda, a um preço acessf́vel ao consumidor e que, ao mesmo tempo, permita uma lucratividade justa e que incentive o crescimento do setor avícola.

O problema consubstancia-se, então, em conhecer os principais fatores que influenciam a produção e o consumo de frango e como eles ocorrem no Brasil, de modo que indique o mais adequado mecanismo que possa conduzir à melhoria da situação alimentar, principalmente das classes de menor poder aquisitivo, ao incremento na disponibilidade interna de alimentos e seja compatível com a elevação do padrão de vida dos avicultores.

O objetivo geral do presente trabalho é o de estudar a oferta e a demanda de frango de corte nu Brasil. Especificamente, visa-se verificar o comportamento da oferta e demanda frente aos preços vigentes no mercado de frango de corte; identificar a relação existente entre a carne de frango e as carnes bovina e surna; detectar a sensihilidade da produção e do consumo em relação a outras variáveis que afetam o mercado de frango de corte, e extrair subsidios que possam sugerir a implementação de políticas mais adequadas ao setor avícola brasileiro.

\section{METODOLOGIA}

O modelo teórico desse trabalho está respaldado nas teorias da oferta e da demanda: $\mathrm{Na}$ lei da oferta, tende a existir uma relação direta 
entre preços e quantidade ofertada no mercado de produto, ao passo que na lei da demanda estabelece-se uma relação inversa entre os preçoș do produto e sua quantidade adquirida.

Associado às curvas de oferta (demanda) estão os coeficientes de elasticidades-preço, que enfocam o grau de sensibilidade de reação dos produtores (consumidores), face às mudanças nos preços do produto.

Com base em procedimentos recomendados por Girão \& Barrocas (1968), ensaiou-se conjuntos alternativos de variáveis como explicativas do fenômeno a analisar, com vistas a selecionar aquele que se apresentasse mais significativo estatisticamente.

A análise das curvas de oferta e demanda foi feita com base na forma funcional Cobb-Douglas, sendo que os coeficientes estimados são as próprias elasticidades.

Da interação entre o conjunto de forças existentes no mercado, resulta, em cada perfodo de tempo, um preço e uma quantidade do produto comercializado ao preço observado. Este $e$ o preço de equilifbrio do mercado, quando então a quantidade oferecida é igual à quantidade demandada. Admite-se que o equilíbrio $\varepsilon$ estável, isto $t$, que qualquer desvio da condição de equilíbrio será temporária e que tanto produtores quanto consumidores têm conhecimento perfeito sobre as condiçōes do mercado e agem num mundo de certeza. $O$ número de produtores é suficientemente grande para que nenhum deles, individualmente, possa afetar o preço de mercado.

As estimativas da oferta e demanda baseiam-se num sistema de equaçōes simultâneas, onde há necessidade de que o preço recebido pelo produtor seja igual ao preço pago pelo consumidor (preço de equilíbrio). Por essa razão, este estudo se utiliza da pressuposição de que o preço da carne de frango no atacado é um "proxy" do preço pago pelo consumidor e do preço recebido pelo avicultor.

O modelo econcmétrico é formado pela equação em que a variável endógena é determinada em furição das variáveis exógenas e endógenas defasadas. Face ao tipo de dados utilizados (series temporais), estarem sujeitos a enviesamento das estimativas dos parâmetros de algumas variáveis, em decorrência de problemas de multicolinearidade, a seleção das variáveis foi processada com base nos pressupostos estatísticos dos modelos, nas variáveis de importância verificadas na literatura e na importância econômica das variáveis predeterminadas consideradas.

Dessa forma, adotou-se o procedimento de selecionar as variáveis que apresentassem coeficientes de regressão parcial estatisticamente significantes, com base nos niveis de correlações parciais, recomendados por Yotopoulos \& Nugent (1976) e Heady \& Dillon (1964).

O método de estimação utilizado foi o de mínimos quadrados em dois estágios (MQ2E) para eliminar o chamado "viés de simultaneidade" 
e conduzir a estimadores consistentes. De acordo com Kmenta (1990) e Intriligator (1978), o problema de um modelo simultâneo é que, geralmente, as equações do sistema incluem variáveis endógenas com explicativas e estas tendem a ser-correlacionadas com os termos de erro. Nestas condições, a aplicação dos mínimos quadrados ordinários (MQO) para estimar os coeficientes conduziria a estimadores tendenciosos e inconsistentes.

Os modelos de análise utilizados foram estimados nos valores observados das variáveis em seus logaritmos. Formalmente, o modelo pode ser expresso pelas equações a seguir:

$\ln Q_{\mathrm{s}}=\ln a_{1}+a_{2} \ln P_{f}+a_{3} \ln P_{r}+a_{4} \ln P_{p}+a_{5} \ln E_{m}+$ $a_{6} \ln Q_{d f}+u_{t}$

$\ln Q d=\ln b_{1}+b_{2} \ln P_{f}+b_{3} \ln P_{b}+b_{4} \ln P_{s}+b_{5} \ln P_{o}+$ $b_{6} \ln R_{p c}+u_{t}$

Onde:

$Q_{s}=Q_{d}$

$Q_{s}=$ produção de frango no ano t, em toneladas;

$P_{f}=$ preço real do quilo do frango no atacado, no ano t, em cruzados;

$P_{r}=$ preço real do quilo da ração balanceada, pago pelo produtor no ano $t$, em cruzados;

$P_{p}=$ preço real do pinto de um dia (unidade), no ano t, em cruzados;

$E_{m}=$ estoque de matrizes, no ans t, em mil cabeças;

$Q_{d f}=$ prociução de frango defasada em um ano, em toneladas;

$P_{b}=$ preço real do quilo de carne bovina no atacado, no ano t, em cruzados;

$P_{s}=$ preço real do quilo e carne suína no atacado, no ano $\mathrm{t}$, em cruzados;

$P_{o}=$ preço real da caixa de 30 dúzias de ovos no atacado; no ano t, em cruzados;

$R_{p c}=$ renda per capita da população no ano $t$, em cruzados;

$u_{t}=$ termo aleatório;

ln = logaritmo neperiano. 
Na relação (1), as variáveis $Q_{s}$ e $P_{f}$ e, na relação (2), as variáveis $Q_{d}$ e $P_{f}$ são endógenas, caracterizando-se um modelo simultâneo, envolvendo as equações de oferta e demanda, respectivamente.

As variáveis ração balanceada, pinto de um dia e estoque de matrizes, no modelo de oferta, constituem-se em insumos indispensáveis à produção avícola; a variável produção defasada em um ano foi incluŕda com a finalidade de se verificar os efeitos das instalaçöes existentes sobre a produção futura; as variáveis preço das carnes bovina e suína e preço do ovo foram inclufdas no modelo de demanda, com o objetivo de detectar relações de substituição e/ou complementaridade com a carne de frango; a variável renda per capita foi introduzida no modelo com a finalidade de se verificar e se analisar o efeito de variaçōes na renda sobre a demanda.

Os dados utilizados referem-se ao perfodo $1972-88$ e foram coletados do Anuário Estatístico do Brasil, Anuário Estatístico da Unidade Central do Sistema de Informação de Mercado Agricola, Boletim Mensal da Divisão de Informações de Mercado, Associação Paulista de Avicultura - APA e Associação Nacional dos Abatedouros Avícolas - ANAB.

As séries referentes a preços foram corrigidas pelo índice Geral de Preços - IGP, Coluna 2, da Fundação Getúlio Vargas (FGV), com base transformada para março de $1986=100$.

As equaçóes de oferta e demanda, identificadas ccin base em procedimentos recomendados por SCHUH (1965), foram consideradas superidentificadas, oferecendo condições de fornecer parámetros estruturais verdadeiros.

\section{RESULTADOS E DISCUSSŌES}

A Tabela 1 apresenta os resultados econometricos do modelo selecionado de cferta e demanda. 


\section{TABELA 1 - ESTIMATIVA DOS PARÂMETROS DO MODELO SELECIONADO DE OFERTA E DEMANDA DE. FRANGO DE CORTE NO BRASIL, PERÍODO 1972-88.}

\begin{tabular}{|c|c|c|c|c|c|c|c|}
\hline \multirow{2}{*}{ Modelo } & \multicolumn{7}{|c|}{ Coeficientes $^{(a)}$} \\
\hline & $C$ & $L P_{f}$ & $L P_{r}$ & $L Q_{d f}$ & $L P_{b}$ & $L P_{s}$ & $L R_{p c}$ \\
\hline Oferta & $\begin{array}{c}-1,467 \\
(-0,588)(b)\end{array}$ & $\begin{array}{c}0,738 * * * \\
(1,984)\end{array}$ & $\begin{array}{l}-0,715^{* * *} \\
(-2,985)\end{array}$ & $\begin{array}{c}1,028 * * * \\
(8,455)\end{array}$ & & & \\
\hline$R=0,984$ & & & & & & & \\
\hline $\begin{array}{l}F^{* * *}(3,12)= \\
D W=1,260\end{array}$ & 317,581 & & & & & & \\
\hline Demanda & $\begin{array}{c}22,713^{* * *} \\
(5,312)\end{array}$ & $\begin{array}{c}-6,106 * * * \\
(-3,615)\end{array}$ & & & $\begin{array}{c}3,969 * * * \\
(2,713)\end{array}$ & $\begin{array}{c}1,224 * \\
(1,355)\end{array}$ & $\begin{array}{l}-2,256^{*} \\
(1,350)\end{array}$ \\
\hline$\ddot{R}^{-2}=0,756$ & & & & & & & \\
\hline $\begin{array}{l}F^{* * *}(4,11)= \\
D W=2,230\end{array}$ & 12,624 & & & & & & \\
\hline
\end{tabular}

Fonte: Dados da pesquisa

(a) $C=$ constante de regressão; $L P_{f}=\log$ do preço real do frango no atacado; $L P=\log$ do preço real da ração balanceada, pago pelo produtor; $L Q_{d f}=\log$ da produçáo de frango defasada em um ano; $L P_{b}=\log$ do preço real da carne bovina no atacado; $L P=\log$ do preço real da carne sufna no atacado $\mathrm{e} L R_{p c}=\log$ da renda per capita da população brasileira.

$b=0$ yalor entre parênteses indica o teste " $t$ ".

* nível de significância entre $10 \%$ e $20 \%$;

** nível de significância de 5\%;

*** nível ae significância de $1 \%$.

Os coeficientes de determinação múltipla corrigidos ( $\dddot{R}^{2}$ ) foram de 0,984 para a oferta e 0,756 para a demanda, indicando, respectivamente, que cerca de $98 \%$ e $76 \%$ da variação total nas quantidades ofertadas e demandadas são explicadas pelas variações nas variáveis inclufdas nas equações de regressão. A estes resultados deve-se considerar os argumentos de Tomek (1973), que questiona a interpretação usual do $R^{2}$ oriundo de estimativas obtidas através dos mf́nimos quadrados em dois estágios.

Utilizou-se o teste de Durbin-Watson para testar as hipoteses nulas de autocorrelação serial nos resíduos calculados. Os valores encontrados 
foram $D W=1,26$ para a oferta e $D W=2,23$ para a demanda. Ambos mostraram-se inconclusivos quanto à presença de autocorrelação serial. Nesse caso, nada se pode afirmar da existência ou não de autocorrelação dos resfduos.

Submetidas as variáveis à análise de correlação simples, foram encontradas alta correlação linear entre as variáveis preço do frango e produção defasada em um ano $(-0,809)$, para a oferta e entre as variáveis preço das carnes de frango e sufna $(0,822)$, para a demanda. Estes problemas de colinearidade, de acordo com Kmenta (1990) e Santos (1972), podem produzir estimativas tendenciosas nos erros padrões, com tendência a aumentá-los influenciando o tamanho dos coeficientes. Mesmo assim, optou-se por mantê-las no modelo, uma vez que são variáveis relevantes no processo produtivo e ainda porque este procedimento encontra respaldo nas afirmativas de Klein (1962), ao dizer que "se a raiz quadrada de $\vec{R}^{2}$ for maior que o coeficiente de correlação, pode-se manter a variável no modelo, sem prejuízo da validade dả análise", o que se verificou no presente trabalho.

As equações de oferta e demanda apresentaram os sinais dos coeficientes das variáveis consistentes com a teoria econômica.

A interpretação econômica dos resultados mostra que a quantidade comercializada (ofertada) de frango pode ser explicada por variações na produção defasada em um ano, no preço da ração balanceada e no preço do próprio produto. E a quantidade demandada de frango pode ser explicada por variações no preço do próprio produto, nos preços das carnes bovina e suína e renda per capita da população.

A elasticidade-preço da oferta para o frango $(0,74)$ é inelástica. Isto implica que os produtores apresentam baixa sensibilidade ao preço do frango. $E$ a elasticidade-preço da demanda $(-6,106)$ e elástica, indicando que os consumidores apresentam alta sensibilidade ao preço do frango e que reduçóes em seus preços, provavelmente, propiciariam aumentos nos seus dispêndios e na receita dos avicultores.

Com relação à renda per capita, a demanda indica que a carne de frango $\varepsilon$ um produto inferior, embora com baixa significância estatística. O estudo de Fernandes et alii (1989) sobre politicas relacionadas com a demanda e a oferta de carnes no Brasil, detectou ser a carne de frango um produto superior, porem, com baixa resposta à renda.

Os coeficientes de elasticidades indicam que, outras coisas permanecendo constantes, uma variação percentual em cada variável independente individualizada, provoca uma variação proporcional na variável dependente (oferta ou demanda). Assim, um aumento de $10 \%$ no preço do frango está relacionado a um incremento de oferta em torno de $7,4 \%$ e uma redução de $61 \%$ na quantidade procurada; acréscimos de $10 \%$ no preço da ração balanceada estão associàdos a uma redução na oferta de 
frango da ordem de 7,2\%; incrementos de $10 \%$ na renda per capita tendem a refletir-se numa redução de $22 \%$ na procura de frango, e aumentos de $10 \%$ no preço da carne bovina estão associados a um aumento em torno de $40 \%$ na procura de frango de corte.

O sinal positivo encontrado para os coeficientes dos preços das carnes bovina e suína (Tabela 1) sugere uma relação de substituição dessas carnes com a carne de frango. Os trabalhos de Silva et alii (1977) e Fernandes et alii (1989) também constataram a possibilidade de substituição entre o frango de corte e as carnes bovina e sufna.

\section{CONCLUSÕES}

As principais conclusões foram as seguintes:

A elasticidade-preço da oferta de frango de corte é inelástica, enquanto que a da demada é elástica.

Verificou-se existir uma relação de substituição entre o frango e as carnes bovina e suína.

As políticas de preços podem ser mais efetivas na melhoria das condições de produção de frango de corte, comparadas com políticas de preços nos insumos básicos.

Polfticas direcionadas ao processo de comercialização, visando a redução das suas margens, tendem a ser mais eficientes, principalmente através de um sistema onde os produtores possam melhor se organizarem.

Políticas de congelamento de preços, a exemplo das implementadas no Brasil a partir de 1986, têm se mostrado ineficientes frente a demandas elásticas.

Uma polf́tica de distribuição de renda poderia ser benéfica ao setor avicola e à populaçāo de menor poder aquisitivo, se implementada através de programas sociais que incluf́ssem o frango na cesta hásica.

\section{BIBLIOGRAFIA}

ANUÁRIO ESTATISTICO DA UNIDADE CENTRAL DO SISTEMA DE INFORMAÇĀO DE MERCADO AGRfCOLA. Braślia, Ministério da Agricultura, 1979/88.

ANUǍRIO ESTATÍSTICO DO BRASIL - 1972/89. Rio de Janeiro, FIBGE, v. 33/49, $1972 / 89$.

A VICULTURA. Mercado Mantém Tendéncia de Alta. Informe-GEPIDESR, Piracicaba, v. 3 n. 9 , p. 16, set. 1990 . p. 12, out. 1990 . 1990. 
BARRIOS, D. L. de A. Aspectos Técnico-Económicos da Avicultura de Postura do Sul de Minas. Informe Agropecudrio, Belo Horizonte, v. 7, n. 79, p. 9-12, jul. 1981.

BOLETIM MENSSAL DA DIVISÄO DE INFORMAÇÕES DE MERCADO. Braślia, Ministério da Agricultura, v. 1/7, n. 1/12, 1972/78.

ENGLERT, S. I. Avicultura; Iudo Sobre Raças, Manejo, Alimentação e Sanidade. Porto Alegre, Centaur 1s, 1974.325p.

FERNANDES, E. de A.; MOUCHREK, E. \& MIRANDA, S. F. de. Aspectos Económicos da A vicultura de Postura. Informe Agropecuário, Belo Horizonte, v. 7, n. 79, p. 3-8, jul. 1981.

FERNANDES, S. G.; PANIAGO, E. \& LIMA, J. E. de. Análise de Alternativas de Polfticas Relacionadas com a Demanda e a Oferta de Carnes no Brasil. Revista de Economia e Sociologia Rural, Brasília, v. 27, n. 4, p.437-61, outJdez. 1989.

GIRAO, J. A. \& BARROCAS, J. M. Análise de Regressão: o Algoritmo Strap. Lisboa, Fundação Calouste Gulbenkian, Centro de Estudos de Economia Agrária, 1968. 52 p.

HEADY, E. O. \& DILLON, J. L. Agricultura Production Function. Ames, Iowa State University, 1964.667 p.

INTRILIGATOR, M. D. Econometric Models, Techniques \& Aplications. Englewood Cliffs, Prentice-Hall, 1978.638 p.

KLEIN, L. R. An Introduction to Econometrics. Englewood Cliffs, Prentice-Hall, 1962. 280 p.

KMENTA, J. Elementos de Econometria; Teoria Econométrica básica. São Paulo, Atlas, 1990. v. $2.711 \mathrm{p}$.

MARRA, R.; MOTA, M. M. \& CONTINI, E. Informaçöes e Índices Búsicos da Economia Brasileira; Subsídios Para os Profissionais das Ciências Sociais Rurais e Para os Gerentes da Pesquisa Agropecuária e Florestal. 4. ed. Brasilia, EMBRAPA-SEA, 1990. 87 p. (EMBRAPA-SEA. Documentos, 01).

PRODUÇÃO, Destinação e Abate de Aves no Brasil: ano 1984. São Paulo, Associação Nacional dos Abatedouros A vicolas, s. d. s. p.

SADIA CONCÓRDIA S/A INDÚSTRIA E COMÉRCIO. Uma Visão de Duas Décadas de Avicultura. Concordia, s. d. n. p.

SANTOS, L. F. dos. Estimativa da Oferta de Arroz, Milho e Feijāo em Minas Gerais. Dissert. (mestr. econ. rural). Viçosa, UFV, 1972.91 p. (MS).

SCHUH, G. E. Curso de Econometria. Viçosa, UFV, 1965.183 p. (Apostila).

SILVA, G. L. S. da; TOYAMA, N. K. \& YOSHII, R. J. Oferta e Demanda de Frangos no Estado de São Paulo. Revista de Econonia Rural, Brasflia, v. 15, p. 193-207, 1977.

TABELAS da Avicultura; Aves 10/89 Ovos. In: ASSOCIAÇÄO PAULISTA DE AVICULTURA. Relatorio Mensal, v. 15, n. 12, n. p. nov. 1989.

TEIXEIRA, M. B. Empresa Rural; Administração, Planejamento, Construçäo. A Granja, Porto Alegre, v. 25, n. 263/64, p. 38/52, dez./jan. $1969 / 70$.

TOMEK, W. G. $R^{2}$ in TSLS and GLS Estimation. American Journal of Agricultural Economics, Lexington, v. 55, n. 4, p. 670, nov. 1973.

YOTOPOULOS, P. A. \& NUGFNT, J. B. Economics, of Development, Empirical Investigations. New York, Harper \& Row, Publishers, 1976. 478 p.

\section{SUMMARY}

\section{POULTRY SUPPLY AND DEMAND IN BRAZIL}

This work was carried out with the objective of studying poultry supply and demand in Brazil. Time series data referring to $1972-1988$ were used. Both supply and demand were analysed according to a mathematical model which was based on the functional form Cobb-Douglas. Parameters were estimated on the basis of two-stage least squares (TSLS). As to supply and demand an econometric model was developed according to a simultaneous equation system. The main results found were as follows: The poultry supply was found to be price inelastic whereas the poultry demand was found to be price elastic; brasilians poultry raisers seem to be more sensitive to the poultry price increases than to price decreases of balanced feed; poultry meat demand to show a substitution relation between the beef and pork and the poultry meat; price "freezing" policies were found to be ineffective in the face of elastic demands. 